\title{
Host response, obesity, and oral health
}

\author{
SYLWIA MAŁGORZATA SŁOTWIŃSKA ${ }^{l}$, ROBERT SEOTWIŃSKI ${ }^{2,3}$ \\ 'Department of Conservative Dentistry, Medical University of Warsaw, Warsaw, Poland \\ ${ }^{2}$ Department of Immunology, Biochemistry and Nutrition, Medical University of Warsaw, Warsaw, Poland \\ ${ }^{3}$ Department of Surgical Research and Transplantology, Mossakowski Medical Research Centre, Polish Academy of Sciences, \\ Warsaw, Poland
}

\begin{abstract}
Proper food choices are part of preventing or reducing the risk of dental caries and periodontal disease. A significant association has been proven between oral diseases and the incidence of systemic diseases. Obesity, just like smoking, is one of the major risk factors for oral disease and is a serious social problem that has reached epidemic proportions in many developed countries. The results of studies on periodontitis confirm the relationship between the values of body mass index (BMI) and the prevalence of periodontal diseases. Adipose tissue is an active endocrine organ and it performs many important functions in the body, such as thermal isolation and protection, storage, and secretion. Many cytokines are secreted proportionally to the amount of fat present and are actively involved in the metabolism of the whole system, including the functioning of the immune system. Therefore, obesity may alter the response of the host to the antigens derived from bacterial plaque, and thus cause disturbances in the inflammatory response in the course of periodontal disease.
\end{abstract}

Key words: obesity, host response, oral health, oral diseases.

(Centr Eur J Immunol 2015; 40 (2): 201-205)

The modern definition of health takes into account human nutrition in the context of the broadly understood promotion of a healthy lifestyle. Health promotion includes the rational choice of diet for both the individual and particular social groups. Proper nutrition of the organism consists of providing a suitable amount of proteins, carbohydrates, lipids, and mineral salts. Proper food choices are part of preventing or reducing the risk of dental caries and periodontal disease. A significant association has been proven between oral diseases and the incidence of systemic diseases. Many patients do not realise the connection between the general state of human health and oral health and diet. Providing clear and accurate information on proper nutrition and a well-balanced diet, as well as a basic knowledge of oral health, will allow patients to understand the importance of a proper diet, not only for the prevention of systemic diseases, but also for the prevention of diseases of the teeth and periodontium. Preventive dentistry consists primarily of adherence to a regimen of daily home oral hygiene and proper eating habits [1]. Consistent implementation of the basic principles of a balanced and optimally matched diet to an individual patient's needs is also very important in this respect, which recommends appropriate consumption of proteins, carbohydrates, and fats as well as products containing minerals and vitamins. The nutrition and quality of the supplied nutrients play a crucial role in the comfort of human life, and have a significant impact on the preventive and health actions, and the course of possible therapeutic intervention. It should be emphasised that both the presence of excess adipose tissue, and its deficiency, may lead to disturbances in the normal regulation of systemic metabolic processes. Failure to comply with the basic dietary principles, and long-term improper nutrition, can lead to pathological conditions involving significant malnutrition or obesity, which in turn has a negative impact on oral health.

Obesity, just like smoking, is one of the major risk factors for oral disease and is a serious social problem that has reached epidemic proportions in many developed countries. In the United States of America, obesity is considered one of the most common causes of death that could prevented $[2,3]$. Improper lifestyle is responsible in $70 \%$ for the risk of obesity, and only $30 \%$ of the risk is attributable to genetic factors $[4,5]$. High blood pressure, high cholesterol, type 2 diabetes, cardiovascular disease, stroke, kidney disease, certain cancers, and diseases of the oral cavity are examples of chronic conditions negatively associated with obesity [68]. Especially dangerous is an early form of obesity that occurs in very young persons, which, if left untreated and unmanaged, deepens into adulthood and leads to serious metabolic and organ disorders [9]. There was a positive correlation found between the presence of periodontal disease and obesity in adults aged 17-21 years [10]. It has been shown that the prevalence of periodontal disease in obese

Correspondence: Sylwia Słotwińska, Department of Conservative Dentistry, Medical University of Warsaw, Miodowa 18, 00-246 Warszawa, Poland, e-mail: sylwia.slotwinska@wum.edu.pl 
young people aged 18-34 years is, by approximately $76 \%$, significantly higher compared with those of the same age with normal body weight [11].

Special anthropometric indicators are applied in the case of obesity diagnosis to assess the degree of the risks of various systemic and general diseases, including periodontal disease $[12,13]$. The most common way to measure obesity is to calculate body mass index (BMI). Body mass index is calculated by dividing weight in kilograms by the square of height in metres. This gives information on the extent of body fat and at the same time the risk of diseases associated with obesity. The results of the studies on periodontitis confirm the relationship between the values of BMI and the prevalence of periodontal diseases [14]. Studies conducted in the United States in subjects with overweight and advanced obesity showed a positive correlation between BMI and parameters estimating the level of oral hygiene and describing the clinical condition of the gingiva and periodontium $[15,16]$. A study conducted in Japan showed a statistically significant correlation between BMI and the depth of periodontal pockets in women with obesity, and demonstrated that BMI above $30 \mathrm{~kg} / \mathrm{m}^{2}$ increased by more than four-fold the risk of periodontitis [17]. Other Japanese studies that analysed the periodontal status using CPITN index (Community Periodontal Index of Treatment Needs), evaluating the periodontal treatment needs, demonstrated a positive correlation between the exacerbation of symptoms indicating significant progression of periodontal disease and the increase in body weight measured by BMI [18].

Other parameters that are used to determine the progress of obesity are an assessment of waist circumference, the ratio of waist to hip circumference, and the measurement of total body fat. There are also works in the literature, providing evidence for the existence of a relationship between the above-listed parameters of the degree of obesity qualification and indicators evaluating the progression of periodontal disease and oral hygiene [19].

Adipose tissue is an active endocrine organ, and it performs many important functions in the body, such as thermal isolation and protection, storage, and secretion. The cells of adipose tissue, called adipocytes, secrete dozens of biologically active molecules, which can significantly modulate reactions occurring in the body. These include leptin, resistin, tumor necrosis factor $\alpha(\mathrm{TNF}-\alpha)$, interleukins: IL-1, IL-6, IL-8, and IL-10, growth factors, complement components, angiotensinogen, plasma plasminogen activator-1 (PAI-1), and a number of other substances. Many cytokines are secreted proportionally to the amount of fat present and are actively involved in the metabolism of the whole system, including the functioning of the immune system. Therefore, obesity may alter the response of the host to the antigens derived from bacterial plaque, and thus cause disturbances in the inflammatory response in the course of the periodontal disease [20,21].
Periodontitis is a disease process that causes progressive destruction of the periodontal tissues, including the loss of gingival connective tissue, destruction of periodontium, and alveolar bone resorption. Aetiological factors are microorganisms derived from plaque, while inflammation is a host response to bacterial antigens. The most commonly isolated bacteria from pathological periodontal pockets in chronic periodontitis diagnosed in patients over 35 years of age are: Porphyromonas gingivalis, Treponema denticola, and Tannerella forsythia, whereas in young people before the age of 35 Aggregatibacter (Actinobacillus) actinomycetemcomitans is the main species found in the periodontal pockets. The latter bacterium has been associated with aggressive periodontitis. Multiple mediators of inflammation and immune response are detected in gingival crevicular fluid during periodontitis [22, 23]. Patients with periodontitis show a significant increase in the levels of IL-6, IL-8, and TNF- $\alpha$ in gingival crevicular fluid. A positive correlation was demonstrated in patients with significant obesity and coexisting periodontal disease between the concentrations of these cytokines in gingival fluid and BMI values. The study by Bruun et al. showed a significant reduction $(25-30 \%, p<0.001)$ in the levels of TNF- $\alpha$ and IL-6 in the peripheral blood serum of obese patients after switching to a calorie-restricted diet [24].

A statistically significant increase in TNF- $\alpha$ in peripheral blood serum has been found in overweight and obese people, which was derived from the accumulated body fat, which could promote the development of periodontal disease or exacerbate the course of already existing disease. Tumor necrosis factor $\alpha$ stimulates fibroblasts to synthesise enzymes degrading extracellular matrix proteins, and osteoclasts to activate bone resorption. The expression of IL-6 starts in adipose tissue, and it is subsequently released into the circulation. It is a cytokine, the elevated serum of which is considered an indicator of increased risk of cardiovascular events, especially in obese individuals. Interleukin 6 is also involved in the mechanisms of uncontrolled gains of body weight in patients with refractory obesity. In the course of periodontitis, IL-6 activates osteoclasts in the process of destruction of lamina dura of alveolar bone and induces periodontal tissue destruction via activation of matrix metalloproteinases, collagenases in particular [25].

Most obese people have abnormal lipid parameters, including mainly significant increase in triglycerides. Hyperlipidaemia is one of the symptoms that are often observed in the course of infectious diseases. A significant positive correlations was found in overweight and obese people between elevated levels of peripheral serum lipids and indicators of periodontitis [26].

Blood vessels of periodontium in obese people have thickenings on the most inner walls, which is a symptom of reduced blood flow. It is believed that this is the effect of action of the PAI-1 - adipokine, the level of which is markedly increased in visceral fat in obese individuals. 
The reduction of blood flow in periodontal vessels is one of the pathogenic mechanisms of the development of periodontal disease [27].

In recent years, a significant increase in the proportion of people suffering from type 2 diabetes among adults has been shown, which is primarily due to increased obesity induced by the decrease in glucose tolerance. The incidence of type 2 diabetes among obese adolescents is increasing significantly. Type 2 diabetes primarily affects obese middle-aged people who have a sedentary life style. It is believed that TNF- $\alpha$ produced by adipose tissue is critical in the pathogenesis of insulin-dependent diabetes mellitus and in the presence of insulin resistance. It is also a common "denominator"/factor linking obesity and periodontitis. Patients with type 2 diabetes are twice as likely to suffer from periodontal disease compared with those without diabetes. Obesity is a risk factor for both type 2 diabetes and periodontal disease. Moreover, diabetes increases the risk of gingivitis. Inflammation induced by pro-inflammatory cytokines produced in adipose tissue, and locally in the case of gingivitis, is a common feature of insulin-dependent diabetes, periodontal disease, and obesity [28].

It is known that obesity lowers insulin sensitivity, which leads to insulin resistance. Giant fat cells - adipocytes - occurring in adipose tissue in obese patients, have a number of metabolic functions, including the regulation of energy levels in the body by the secretion of biologically active molecules and hormones [29]. One of them is leptin - a hormone produced by fat cells, which plays a key role in regulating body weight. Leptin increases fat storage, reduces appetite, and is one of the inductors of insulin secretion by the pancreas. The concentration of leptin in the blood plasma of obese people is significantly increased due to the storage of large amounts of adipose tissue, which in turn causes a lack of the suppressive action of leptin on appetite. This paradox is known as leptin resistance. Obese patients can be characterised by two states of resistance: insulin resistance and leptin resistance [30]. These characteristics must be complemented with a very high risk of periodontal disease and gingivitis as well as increased risk of incidence of dental caries [31-33].

Dental caries is a chronic pathological condition, attacking mineralised structures of teeth, with a multifactorial but well understood aetiology. The main link in the aetiological chain of dental caries is obviously cariogenic microorganisms from dental plaque, mainly Streptococcus mutans group, in combination with carbohydrate diet. The passage of time is required, so that the demineralising action of the acid, which is a product of sugar metabolism by bacteria, can injure the hard tissue structure of the tooth. Various additional parameters should also be included, of which the most important are dietary habits and socioeconomic and demographic factors. Numerous studies exist, both clinical and experimental, concerning dental caries.
However, little is known about the relationship of caries with obesity. The available literature shows that obesity is certainly associated with the occurrence of early childhood and puberty caries. Modéer et al. demonstrated that children/adolescent obesity (age of patients: 10-17 years) was significantly correlated $(p<0.001)$ with the surface of teeth affected by caries as well as plaque and gingivitis indices [34]. Studies carried out in adults with severe overweight, obesity, and coexisting diabetes indicate a significantly higher frequency of caries in these patients compared to the control group, i.e. people with diabetes without obesity $[35,36]$. However, it is difficult to clearly ascertain whether this is only due to the significant overweight or diet, nutrition, and hygiene negligence, which are often caused by an extremely complex and difficult to diagnose psychological and image situation, in which obese adult patients often find themselves.

A separate discussion should be dedicated to so-called metabolic syndrome (MS), which is very often diagnosed in patients with overweight and obesity. This syndrome, also know as insulin resistance syndrome, is caused by both genetic and environmental factors, of which the most important are the lack of physical activity and excessive food intake [37-39]. There are several definitions concerning this disease entity, provided by the World Health Organisation (WHO 1998), the International Diabetes Federation (IDF 2006), the European Group for the Study of Insulin Resistance (EGIR 2002), and several other medical organisations. These definitions possess several common biological and clinical features, which as a consequence of chronic synergistic action lead to serious systemic disorders. The clinical diagnosis of MS is based on the identification of three of the five recognised criteria, including mandatory abdominal obesity. These are: abdominal obesity - for women (waist circumference) $>88 \mathrm{~cm}$; for men (waist circumference) $>102 \mathrm{~cm}$; elevated triglyceride levels $>150 \mathrm{mg}$; reduced HDL cholesterol $<50 \mathrm{mg}$ for women and $<40 \mathrm{mg}$ for men; glucose in blood glucose on an empty stomach $>100-110 \mathrm{mg}$; and high blood pressure $>130 / 85 \mathrm{~mm} \mathrm{Hg}$. It has been shown that individuals diagnosed with MS are significantly more prone to caries and periodontitis compared with the reference group. What is more, a statistically significant positive correlation was found between the diagnostic criteria of metabolic syndrome and clinical parameters estimating the severity of periodontal disease [40].

The severe and often irreversible effects of obesity on health and the mental condition of the obese person indicate that studies on a possible association of obesity with many systemic and oral diseases are extremely important and necessary. Obesity has a negative impact on both general health and the health of the oral cavity. Promoting healthy eating behaviour and appropriate physical activity are fundamental elements of modern prophylaxis of periodontal and teeth diseases and prevention or reduction of 
obesity. Highly important is the selection of adequate nutrients, in relation to the age group, health status, physical activity, and the type of work performed by the person for whom the diet is designed. Nutrients should be consumed in adequate quantities and proportions. Restriction of carbohydrates and a reduction in fat intake cause not only a significant decrease in the incidence of dental caries and a significant improvement in clinical condition of periodontium, but also considerably affect the processes that reduce body weight. Children from an early age should be provided with a diet rich in minerals and vitamins, which directly affects the proper development of teeth and periodontal tissues and is of great importance in preventive dentistry. School meals for children in preschool and school age should contain all the recommended nutrients: first of all milk, cheese, cottage cheese, lean meat, fish, eggs, fresh vegetables, and fruit. It should be ensured that meals are eaten at least five times a day. Regular drinking of milk and dairy products and increased consumption of fruits, vegetables, and whole grains is beneficial to the clinical condition of the teeth, periodontium, and oral mucosa. Similar recommendations should also be addressed to adults. Another important element of proper nutrition for children and adults is to reduce frequent eating between meals, called snacking. This particularly applies to sweet snacks.

A properly constructed diet and adequate nutrition are some of the most important factors conditioning normal development of the entire stomatognathic system, primarily including the morphological and histological structure of the teeth and specific structure of periodontal tissue, which in combination with daily and regular oral hygiene will help to maintain healthy teeth and periodontium for a long time.

\section{The authors declare no conflict of interest.}

\section{References}

1. Moynihan P, Petersen PE (2004): Diet, nutrition and the prevention of dental diseases. Public Health Nutr 7 (1A): 201226.

2. Flegal KM, Carroll MD, Kit BK, Ogden CL (2012): Prevalence of obesity and trends in the distribution of body mass index among US adults, 1999-2010. JAMA 307: 491-497.

3. Flegal KM, Kit BK, Graubard BI (2014): Body mass categories in observational studies of weight and risk of death. Am J Epidemiol 180: 288-296.

4. Meinz D (2004): Nutrition, diet and dentistry today. Gen Dent 52: 387-389.

5. Al-Zahrani MS, Borawski EA, Bissada NF (2005): Periodontitis and three health-enhancing behaviors: maintaining normal weight, engaging in recommended level of exercise and consuming a high-quality diet. J Periodontol 76: 1362-1366.

6. Al-Zahrani MS, Bissada NF, Borawski EA (2005): Diet and periodontitis. J Int Acad Periodontol 7: 21-26.
7. Bawadi HA, Khader YS, Haroun TF, et al (2011): The association between periodontal disease, physical activity and healthy diet among adults in Jordan. J Periodontal Res 46: 74-81.

8. Dahiya P, Kamal R, Gupta R (2012): Obesity, periodontal and general health: relationship and management. Indian J Endocrinol Metab 16: 88-93.

9. de Castilhos ED, Horta BL, Gigante DP, et al. (2012): Association between obesity and periodontal disease in young adults: a population-based birth cohort. J Clin Periodontol 2012; 39: 717-724.

10. Reeves AF, Rees JM, Schiff M, Hujoel P (2006): Total body weight and waist circumference associated with chronic periodontitis among adolescents in the United States. Arch Pediatr Adolesc Med 160: 894-899.

11.Al-Zahrani MS, Bissada NF, Borawskit EA (2003): Obesity and periodontal disease in young, middle-aged, and older adults. J Periodontol 74: 610-615.

12. Wood N, Johnson RB, Streckfus CF (2003): Comparison of body composition and periodontal disease using nutritional assessment techniques: NHANES III. J Clin Periodontol 30: 321-327.

13. World Health Organization (WHO) (1997): Oral Health Surveys: Basic Methods. 4th ed. World Health Organization, Geneva; 6-39.

14. Benguigui C, Bongard V, Ruidavets JB, et al. (2012): Evaluation of oral health related to body mass index. Oral Dis 18: 748-755.

15.Ogden CL, Carroll MD, Kit BK, Flegal KM (2013): Prevalence of obesity among adults: United States, 2011-2012. NCHS Data Brief 131: 1-8.

16.Ogden CL, Carroll MD, Kit BK, Flegal KM (2014): Prevalence of childhood and adult obesity in the United States, 2011-2012. JAMA 311: 806-814.

17. Saito T, Shimazaki Y, Kiyohara Y, et al. (2005): Relationship between obesity, glucose tolerance, and periodontal disease in Japanese women: the Hisayama study. J Periodontal Res 40: 346-353.

18. Saito T, Shimazaki Y, Sakamoto M (1998): Obesity and periodontitis. N Engl J Med 339: 482-483.

19. Ngatia EM, Gathece LW, Macigo FG, et al. (2008): Nutritional and oral health status of an elderly population in Nairobi. East Afr Med J 85: 378-385.

20.Esposito K, Giugliano G, Scuderi N, Giugliano D (2006): Role of adipokines in the obesity - inflammation relationship: the effect of fat removal. Plast Reconstr Surg 118: 1048-1057.

21.Zeyda M, Stulnig TM (2007): Adipose tissue macrophages. Immunol Lett 112: 61-67.

22. Genco RJ (1996): Current view of risk factors for periodontal diseases. J Periodontol 67: 1041-1049.

23. Williams RC (1990): Periodontal disease. N Engl J Med 322: 373-382.

24. Bruun JM, Stallknecht B, Helge JW, Richelsen B (2007): Interleukin-18 in plasma and adipose tissue: effects of obesity, insulin resistance, and weight loss. Eur J Endocrinol 157: 465471.

25.Bruun JM, Verdich C, Toubro S, et al. (2003): Association between measures of insulin sensitivity and circulating levels of interleukin-8, interleukin-6 and tumor necrosis factor-alpha. Effect of weight loss in obese men. Eur J Endocrinol 148: 535-542. 
26. Saito T, Shimazaki Y, Koga T, et al. (2001): Relationship between upper body obesity and periodontitis. J Dent Res 80 : 1631-1636.

27. Saito T, Shimazaki Y (2007): Metabolic disorders related to obesity and periodontal disease. Periodontol 2000 43: 254266.

28. Khader Y, Khassawneh B, Obeidat B, et al. (2008): Periodontal status of patients with metabolic syndrome compared to those without metabolic syndrome. J Periodontol 79: 20482053.

29. Wisse BE (2004): The inflammatory syndrome: the role of adipose tissue cytokines in metabolic disorders linked to obesity. J Am Soc Nephrol 15: 2792-2800.

30. Bastard JP, Maachi M, Lagathu C, et al (2006): Recent advances in the relationship between obesity, inflammation, and insulin resistance. Eur Cytokine Netw 17: 4-12.

31.Pischon N, Heng N, Bernimoulin JP, et al. (2007): Obesity, inflammation, and periodontal disease. J Dent Res 86: 400-409.

32. Riley M (2007): Incorrect nutrition as a risk factor for periodontal disease. Alpha Omegan 100: 85-88.

33. Willerhausen B, Blettner M, Kasaj A, Hohenfellner K (2007): Association between body mass index and dental health in 1,290 children of elementary schools in a German city. Clin Oral Investig 11: 195-200.

34. Modéer T, Blomberg CC, Wondimu B, et al. (2010): Association between obesity, flow rate of whole saliva, and dental caries in adolescents. Obesity 18: 2367-2373.

35. Timonen P, Niskanen M, Suominen-Taipale L, et al. (2010): Metabolic syndrome, periodontal infection, and dental caries. J Dent Res 89: 1068-1073.

36.Levine R (2012): Obesity and oral disease - a challenge for dentistry. Br Dent J 213: 453-456.

37. Vega GL (2004): Obesity and the metabolic syndrome. Minerva Endocrinol 29: 47-54.

38. Grundy SM (2006): Does the metabolic syndrome exist? Diabetes Care 29: 1689-1696.

39. Eckel RH, Alberti KG, Grundy SM, Zimmet PZ (2010): The metabolic syndrome. Lancet 375: 181-183.

40. Kwon YE, Ha JE, Paik DI, et al. (2011): The relationship between periodontitis and metabolic syndrome among a Korean nationally representative sample of adults. J Clin Periodontol 38: 781-786. 